\title{
Gas erleuchtet die Schweiz. Stadtgas als Energieträger des 19. und frühen 20. Jahrhunderts
}

\author{
Maximilian Oswald \\ Kerngebiet: Wirtschafts- und Sozialgeschichte \\ eingereicht bei: Univ.-Prof. Dr. Patrick Kupper \\ eingereicht im Semenster: WS 2015/2016 \\ Rubrik: SE-Arbeit
}

\begin{abstract}
Gas Illuminates Switzerland. Town Gas as an Energy Carrier of the $19^{\text {th }}$ and early $20^{\text {th }}$ century

This paper describes history and focuses on the development of town gas in Switzerland and its biggest city Zurich. The problems and the challenges the gas industry had to face during its existence - especially concerning the establishment of electricity as a competitor on the energy market - will thoroughly be discussed.
\end{abstract}

\section{Einführung}

Man betätigt einen Hebel und schon ist der Raum mit Licht durchflutet - bereits seit langer Zeit eine absolute Selbstverständlichkeit. Dasselbe gilt für Warmwasser oder Kochstellen. Wie groß die Abhängigkeit von diesen alltäglichen Dingen ist, bemerkt man erst dann, sobald eines der Genannten plötzlich nicht mehr auf Knopfdruck reagiert. Wenn etwa der Boiler seinen Dienst quittiert und man sodann auf die heiße Dusche verzichten muss.' Den Tag in Folge ohne Licht oder Warmwasser bestreitend, stellt sich vielleicht die Frage, seit wann es diesen Luxus des "Schalter hoch - Licht/ Wärme an" denn bereits gibt.

Die Antwort findet sich, wenn man sich mit der Geschichte des Stadtgases auseinandersetzt. Mit dem Anschluss von Privathäusern an das Gasnetz ergab sich erstmals

1 Der Autor schreibt hier aus eigener Erfahrung. 
für eine große Bevölkerungsschicht die Möglichkeit, durch Betätigung eines simplen Hebels „Energie“ in die eigenen vier Wände fließen zu lassen - anfangs vornehmlich zur Beleuchtung, später auch zu Heiz- und Kochzwecken. Stadtgas war in den industrialisierten Staaten ein zentraler Energieträger des 19. und 20. Jahrhunderts, obgleich das immer mehr in Vergessenheit zu geraten scheint. Ehemalige Gaswerke sind in heutigen Tagen v.a. als Veranstaltungszentren² bekannt, mit ihrer Geschichte sind wohl nur noch wenige vertraut. Dieser Umstand macht es auch so überaus lohnenswert, sich mit diesem Sujet auseinanderzusetzen.

In der vorliegenden Arbeit wird die Entwicklung des Stadtgases in der Schweiz von seinen Anfängen bis zum Ende des Ersten Weltkrieges Thema sein. Zunächst wird eine kurze Zusammenfassung der Geschichte dieses Energieträgers von seiner Entdeckung bis hin zu seiner Ausbreitung in Westeuropa dargelegt. Im Anschluss folgt die Ausführung zum Werdegang des Stadtgases in der Schweiz, wobei den Gaswerken der Stadt Zürich zur exemplarischen Darstellung ein eigenes Unterkapitel gewidmet wird.

Zentrale Fragen, die auf den folgenden Seiten beantwortet werden sollen, sind, wie sich die Etablierung des Stadtgases in der Schweiz und in Zürich im Speziellen gestaltet hat und mit welchen Problemen bzw. Herausforderungen die Gaswirtschaft im Laufe ihres Bestehens zu kämpfen hatte - insbesondere bezogen auf das Aufkommen von elektrischem Strom als konkurrierender Energiequelle. Zu vermuten ist, dass die Gasindustrie der Schweiz als innovativer und junger Wirtschaftszweig sich schnell auf Veränderung in Angebot und Nachfrage einstellen konnte. Diese These gilt es auf den folgenden Seiten zu belegen.

Als Quellengrundlage dienen hierzu zeitgenössische Artikel des technischen Fachmagazins „Schweizerische Bauzeitung”. Darin lässt sich etwa der damals aktuelle Diskurs rund um die Konkurrenz zwischen Stadtgas und Elektrizität gut nachvollziehen. Ergänzend stehen zwei fachspezifische Dissertationen zur Verfügung, in denen sich die Autoren eingehend mit Kohle bzw. Kohlegas in der Schweiz auseinandergesetzt haben. Abgerundet wird das Bild durch ausgewählte Sekundärliteratur, etwa David Gugerlis bekannte Monographie „Redeströme”.

\section{Stadtgas - Die kurze Geschichte eines Energieträgers}

\section{Die Anfänge}

Als ein möglicher Entdecker der Leuchtgas-Gewinnung gilt der britische Geistliche und Naturforscher John Clayton. Im Jahr 1688 soll er durch Zufall den „Spirit of Coal” destilliert haben - so teilte er es seinem Kollegen Robert Boyle in einem Brief mit. Berichte von Bachwasser, das leicht entzündlich gewesen sein soll, erregten seine Neugier. Um diesem Phänomen auf den Grund zu gehen, hatte er einen Gehilfen entsandt, der Kohle als den möglichen Urheber ausmachen konnte. In seinem Labor begann 
Clayton dann, die Kohle in einem Retortenofen zu destillieren. Zunächst entstand ein schwarzes Öl und zu guter Letzt ein Gas (spirit), das sich auf keine Weise kondensieren ließ. Er fing das Gas in Tierblasen auf und konnte es darin beliebig lange aufbewahren und nach Bedarf anzünden, wobei es ein helles Licht erzeugte. ${ }^{3}$

Wie es scheint, war es Clayton und Boyle nur im Ansatz bewusst, welche Entdeckung hier gemacht worden war, denn einer breiteren wissenschaftlichen Öffentlichkeit wurde das Phänomen erst rund fünfzig Jahre später vorgestellt, als es die Royal Society 1739 schließlich in ihren „Philosophical Transactions” publizierte. Dennoch blieb das Gas noch für das restliche Jahrhundert als Energieträger weitgehend ungenutzt und wurde vielmehr zu Unterhaltungszwecken („brennbare Luft“) verwendet. Seinen Siegeszug als moderne Lichtquelle konnte es dann ab etwa 1800 feiern. Um die neuartigen und in immer größerer Zahl entstehenden Industrieanlagen mit ausreichend Beleuchtung versorgen zu können, bediente man sich vermehrt des Gases als Mittel der Wahl. Die Etablierung dieses im Grunde schon über hundert Jahre alten Energieträgers folgte somit Hand in Hand mit der fortschreitenden Industrialisierung. ${ }^{4}$

Erster Nutzer des Leuchtgases war das Unternehmen Watt \& Boulton, ${ }^{5}$ das in Birmingham angesiedelt war. Der dort beschäftigte Ingenieur William Murdoch war bei der Entwicklung der Leuchtgas-Anlagen federführend. In mehreren Versuchsreihen beschäftigte er sich sowohl mit der Gasherstellung selbst, als auch mit dessen Aufbewahrung bzw. Weiterleitung zu den Lampen. Schon bald verwarf er transportable Lösungen, wie jene der Tierblasen-Gaslampe von Clayton. Vom Ort der Produktion sollte das Gas mittels Röhren zu einem Speicher (Gasometer) und von dort zu den einzelnen Verbrauchern geleitet werden. Reguliert wurde dieses System mittels Ventilen. Aus experimentellen Vorläufermodellen wurden nach und nach professionelle, für die Industrie brauchbare Anlagen entwickelt. 1808 veröffentlichte Murdoch seine gewonnenen Erkenntnisse schließlich ebenso in den "Philosophical Transactions" der Royal Society - knapp siebzig Jahre nach den Ausführungen von Clayton. Die darin beschriebene Anlage sollte grundlegend für alle späteren Gasanstalten werden. ${ }^{6}$

\section{Expansion}

In den Anfängen stand Leuchtgas nur der Industrie zur Verfügung. In Großbritannien gab es auch keine Bestrebungen, dies zu ändern. Der erste nennenswerte Versuch, Gas etwa auch in Privathaushalten anzuwenden, kam aus Frankreich. Der Ingenieur Philippe Lebon entwickelte um 1800 das Konzept einer Thermolampe, die beleuchten und zugleich auch heizen sollte. Das Potential dieser Erfindung wurde jedoch nicht erkannt, es blieb bei einer Versuchsanlage in Lebons Privathaus.?

3 Kenneth Hutchinson, The Royal Society and the Foundation of the British Gas Industry, in: Notes and Records of the Royal Society of London 39 (1985), No. 2, S. 245-270, hier S. 245; Wolfgang Schivelbusch, Lichtblicke. Zur Geschichte der Helligkeit im 19. Jahrhundert, Frankfurt am Main 2004, S. 23. Schivelbusch, Lichtblicke, S. 23 f.

Zurückgehend auf James Watt, "Erfinder" der Dampfmaschine.

Schivelbusch, Lichtblicke, S. 25 f.

Ebd., S. 27-31. 
Seinen Siegeszug im privaten Raum startete das Leuchtgas dann schließlich wiederum in England. Schon wenige Jahre nach Lebons Experimenten wurde die erste kommerzielle Gasgesellschaft gegründet - namentlich die "Light and Coke Company" 1810 in London. ${ }^{8}$ Dem vorausgegangen waren umfangreiche Werbemaßnahmen, die potentielle Kunden mit der neuen Technologie vertraut machen sollten. Die Rechnung ging auf, die Gasgesellschaft war profitabel und Vorbild für weitere Unternehmungen. Entscheidend dabei war auch, dass die Konsumenten mittels Leitungsrohren vom zentralen Gaswerk aus versorgt werden konnten. Modell hierfür war das bereits bestehende Netz der Wasserversorgung, das in der Stadt teilweise schon seit dem frühen 18. Jahrhundert vorhanden war. ${ }^{9}$ London war, was die Expansion der Gaswerke und ihrer Netze betrifft, Vorreiter in Europa. Schnell etablierten sich neue Gesellschaften und die Versorgungsdichte wuchs an. Aber auch andere englische Kommunen zogen bald nach. Bereits Mitte der 1820erJahre verfügten die meisten großen Städte über eigene Gaswerke, im Laufe der folgenden Jahrzehnte konnte man sogar in Kleinstädten und gar Dörfern Straßen und Gebäude mit Gas erleuchten. ${ }^{10}$

Während sich diese neue Technologie rasch in Großbritannien ausbreitete, hinkte das Festland-Europa noch etwas hinterher. In Frankreich etwa scheiterten die ersten Gasgesellschaften allesamt an der zu geringen Nachfrage. Erst Ende der 1820er-Jahre waren vereinzelt mit Gasbeleuchtung ausgestattete öffentliche Straßen und Plätze vorzufinden - ein nennenswerter Ausbau in Paris streckte sich aber bis in die 1840er-Jahre hinein. Auch im deutschsprachigen Raum benötigte es einige Jahre, bis die Gastechnologie flächendeckend genutzt wurde. ${ }^{11}$ Wien bekam seine erste Gasbeleuchtungsanstalt im Jahre 1829,12 in Berlin ${ }^{13}$ wurden bereits drei Jahre früher Straßen mit Gas erhellt, Bern folgte als erste eidgenössische Stadt 1842. ${ }^{14}$ Am Ausbau der Gasversorgung war hier insbesondere das britische Unternehmen „Imperial Continental Gas-Association" (ICGA) maßgeblich beteiligt. ${ }^{15}$

\section{Funktionsweise von Gaswerken}

Wie in vielen anderen industriellen Fertigungsprozessen führten technische Neuerungen auch hier zu einer recht schnell fortschreitenden Optimierung der Gasherstellung. Je nach Zeit und Standort bediente man sich unterschiedlicher Produktionsvarianten

8 Hans von Jüptner, Wärmetechnische Grundlagen der Industrieöfen. Eine Einführung in die Wärmelehre und gedrängte Übersicht über die verschiedenen Arten von Brennstoffen und ihre Verwertung, Berlin 1927, S. 207; The National Archives, Chartered Gas Light and Coke Company, O. D. [http://discovery.nationalarchives.gov.uk/ details/rd/3c478014-712e-43c6-8786-c3b871813664], eingesehen 2.12.2015.

9 Schivelbusch, Lichtblicke, S. $31 \mathrm{ff}$.

10 Ebd., S. $36 \mathrm{f}$.

11 Ebd., S. $37 \mathrm{f}$

12 Helmut Kretschmer, Gasometer in Wien. Industrie- und Technikdenkmale im Wandel der Zeit (Wiener Geschichtsblätter Beiheft 1/2001), Wien 2001, S. 8.

13 Gasversorgungsunternehmen in Berlin, Findbuch. Landesarchiv Berlin, Bestandsgruppe A Rep. 259, [http://www. landesarchiv-berlin.de/php-bestand/arep259-pdf/arep259.pdf], eingesehen 1.11.2015.

14 Karl Grunder, Die Kunstdenkmäler des Kantons Zürich. Band IX Der Bezirk Dietikon (Die Kunstdenkmäler der Schweiz 88), Basel 1997, S. 219.

15 Schivelbusch, Lichtblicke, S. 38. 
und auch Ausgangsstoffe. Das grundlegende Herstellungsprinzip war und ist aber im Großen und Ganzen dasselbe. Exemplarisch wird hier eine Form der Stadtgaserzeugung mittels Steinkohle dargestellt - beschrieben im zeitgenössischen Werk "Das Gas als Leucht-, Heiz-, und Kraftstoff in seinen verschiedenen Arten als Steinkohlegas, Holzund Torfgas, Oelgas, Wassergas" aus dem Jahr 1896:

Das Ausgangsprinzip der Stadtgaserzeugung ist die Möglichkeit, Kohle zu verkoken. In Retorten wird die Kohle so weit erhitzt, dass sich dabei die vergasungsfähigen Bestandteile herauslösen und jene dann über ein Aufsteigrohr aus der Retorte ausweichen können. Zurück bleibt die entgaste Kohle in Form von Koks, das u.a. für Heizzwecke weiterverwendet werden kann. In der angeschlossenen sogenannten Vorlage erfolgt durch Kondensation die erste Scheidung des Gases von dampfförmigen Elementen. Daraufhin wird das Gas in einen Kondensator geleitet, in dem eine weitere Abkühlung und Reinigung passiert. Um schließlich noch Bestandteile mit niedrigem Siedepunkt abtrennen zu können, wird das Gas durch Schichten von lockerem Material, etwa Koks, geleitet, woran es diese Verunreinigungen abscheiden kann. Anschließend müssen aus dem Rohgas noch Ammoniak, Schwefelwasserstoff und Kohlensäure herausgelöst werden. Dies geschieht großteiles mittels Bindung an andere chemische Stoffe. Das gereinigte Gas wird zu guter Letzt in einem Gasometer gesammelt und dort zwischengelagert. Diese Gasometer können ihr Volumen je nach eingelagerter Gasmenge vergrößern bzw. verkleinern und stehen unter leichtem Überdruck, um das Gas dann in die angeschlossenen Verteilersysteme weiterzuleiten. Nebenprodukte wie Koks, Teer und Gaswasser ${ }^{16}$ können entweder seitens des Gaswerks selbst verwertet oder gewinnbringend weiterverkauft werden. ${ }^{17}$

\section{Gas erleuchtet die Schweiz}

\section{Stadtgas in der Schweiz}

Die Schweiz war, wie zuvor schon erwähnt, erst recht spät in die Gasproduktion eingestiegen. ${ }^{18}$ Als erste Stadt erhielt Bern im Jahr 1842 ein Gaswerk, es folgten Genf 1844, Lausanne vier Jahre darauf und Basel 1851. Betrieben wurden die Anlagen anfangs wie auch in anderen europäischen Staaten üblich - von privaten oder halböffentlichen Unternehmen, die von den jeweiligen Kommunen Konzessionen erhalten hatten. Zumeist waren darin die für die städtische Beleuchtung notwendigen Gasabgabemen-

16 Bzw. das darin enthaltene Ammoniak.

17 Otto Pfeiffer, Das Gas als Leucht-, Heiz- und Kraftstoff in seinen verschiedenen Arten als Steinkohlegas, Holz- und Torfgas, Oelgas, Wassergas. Fabrikation und Verwendung nach dem neuesten Standpunkt, unter Berücksichtigung der Konkurrenzverhältnisse zwischen Gas und Elektrizität, Zum 100-jährigen Jubiläum der Gasindustrie, Weimar 1896, S. 61-63.

18 Dezidierte Gründe hierfür waren in der eingesehenen Forschungsliteratur nicht zu finden. Ob die Minderwertigkeit der heimischen Kohlereserven und der mühsame Import von Kohle aus deutschen und französischen Lagerstätten lange Zeit abschreckend auf Unternehmer und Städte wirkten, wäre nur eine von vielen Vermutungen, die dazu angestellt werden können. 
gen geregelt. „Überschüssiges“ Gas konnte an Private verkauft werden - wobei dieser Teilbereich anfangs noch einen untergeordneten Rang einnahm. ${ }^{19}$

Während diese Eigentümerstrukturen zum einen über gewisse Vorteile verfügte, so hatte die Gesellschaft für die Errichtung der nötigen Anlagen und Infrastruktur zu sorgen, waren die Kommunen auch mit einigen Nachteilen konfrontiert - etwa wenn es darum ging, Kapazitäten zu erhöhen oder Neuinvestitionen zu tätigen. Hier stießen private Gasversorger schnell an ihre Grenzen. Zudem waren in den Verträgen zwischen Unternehmen und Stadt auch schon oftmals Rückkaufvereinbarungen enthalten, beispielsweise nach Ablauf eines zeitlich begrenzten Monopols. ${ }^{20}$ Somit gingen die meisten Gaswerke um die Jahrhundertwende schließlich in den Besitz der öffentlichen Hand über. ${ }^{21}$

Da die Schweiz selbst kaum über geeignete Kohlevorkommen verfügte bzw. die Verwendung der heimischen Ressourcen oftmals unrentabel war, waren die Gaswerke schnell auf Importe aus Deutschland und Frankreich angewiesen. ${ }^{22}$ So ist es auch nicht verwunderlich, dass der (Aus-)Bau der Gasversorgung eng an den (Aus-)Bau der Eisenbahn-Verbindungen gekoppelt war, die essentiell für einen kostengünstigen Transport der Kohle waren. ${ }^{23}$

\section{Lieferschwierigkeiten erzeugen Krisen}

Die Etablierung des Stadtgases in der Schweiz war keine Geschichte sich aneinanderreihender "Erfolge". Namentlich zwei große Krisen, die folgend kurz beschrieben werden, hatten die Gasproduzenten zu überstehen.

\section{Der Deutsch-Französische Krieg 1870/71}

Der Ausbruch des Krieges zwischen Preußen und Frankreich bedeutete für die schweizerische Gasindustrie eine substantielle Herausforderung. Wie schon zuvor beschrieben, war die Schweiz von Kohleimporten aus diesen beiden Ländern in hohem Grade abhängig. Schon bald war klar, dass es insbesondere bei den Transportkapazitäten zu Engpässen kommen würde. Das preußische Militär benötigte die Eisenbahn für ihre Truppenbewegungen, die für den Kohletransport erforderlichen Lokomotiven und Waggons standen nicht mehr zur Verfügung. Zudem wurde über das Saarland - das wichtigste Kohlerevier für die Schweiz - ein Ausfuhrverbot verhängt, das schließlich jedoch nur etwa einen Monat Bestand hatte. An der prekären Lage des Transports änderte sich indes nichts, sodass die Schweiz selbst Züge in das Saarland entsenden musste. Eine verlässliche Versorgung konnte aber nicht hergestellt werden. Während es Groß-

19 Daniel Marek, Kohle. Die Industrialisierung der Schweiz aus der Energieperspektive 1850-1900, phil.-hist. Diss. Bern 1992, S. 105; Hans Peter Langbein, Die schweizerische Steinkohlengasindustrie an der Schwelle des Atomzeitalters, phil.-hist. Diss. Basel 1961, S. 36 ff.

20 Marek, Kohle, S. 105 f.

21 Ebd.

22 Langbein, Steinkohlegasindustrie, S. 46.

23 Grunder, Kunstdenkmäler, S. 219; Marek, Kohle, S. 108. 
abnehmern durch bestehende Handelsverträge noch eine Zeit lang möglich war, Kohle zu verträglichen Preisen zu importieren, spürten Kleinverbraucher und Zwischenhändler schon bald die Lieferengpässe. Die Reserven waren schnell aufgebraucht und die Konsumenten griffen sodann gezwungenermaßen auf heimische Rohstoffe, wie etwa Brennholz, zurück. Zudem stiegen die Importe aus französischen Kohlegruben. ${ }^{24}$

Während andere Sparten auf Alternativen ausweichen konnten, traf die Kohlennot die Gaswerkbetreiber im besonderen Maße. Zwar war die Saarkohle bereits bestellt und abholbereit, es scheiterte aber am Abtransport, da die Kapazitäten dafür fehlten. Die Verwendung minderwertiger Kohle und eine Drosselung der Gaslieferungen waren die Folge.

Aber auch nach dem Krieg blieb die Lage weiter angespannt. Die Depots mussten wieder aufgefüllt werden, um den Kunden eine verlässliche Energieversorgung gewährleisten zu können. Die Kohlepreise stiegen jedoch noch bis in das Jahr 1873 hinein weiter an, um sich erst darauf wieder auf Vorkriegsniveau einzupendeln. ${ }^{25}$

Die Streikwelle 1898 bis 1991

Die 1880er-Jahre waren geprägt von einer steigenden Konjunktur und damit auch von einer wachsenden Nachfrage nach Kohle. 1889 begannen jedoch auch die ersten Streiks in deutschen Kohlebergwerken. Vom Rheinland breiteten sie sich bis zu den für die Schweiz so wichtigen Saargruben aus. Im Mai legten die Arbeiter, die sich in Komitees zusammengeschlossen hatten, ihr Gerät nieder, um gegen die herrschenden Bedingungen zu protestieren. Nachdem im Dezember Verhandlungen zwischen Grubendirektion und Belegschaft gescheitert waren, traten die Kumpel abermals in Streik. Die daraus resultierenden Produktionsausfälle - gepaart mit dem generell steigenden Kohlebedarf und der Tatsache, dass die Deutsche Reichsbahn vermehrt Kohlereserven einlagerte - führten dazu, dass die schweizerischen Abnehmer abermals einen Rückgang der Kohlelieferungen hinnehmen mussten. Die Großverbraucher reagierten mit dem Import von französischer Kohle und dem Horten von Reserven, woraufhin der Preis weiter anstieg.

Diese Entwicklungen ließen die Saarkohle nach und nach an Bedeutung verlieren - auch, weil das Deutsche Reich selbst seinen Bedarf mit diesem heimischen Rohstoff decken wollte. Andere Kohlen hatten jedoch eine für die Gasproduktion mindere Qualität, weshalb auch größere Mengen davon verwendet werden mussten. ${ }^{26}$ Die Lage entspannte sich erst wieder im Laufe des Jahres 1891, als die erschöpften Kohledepots wieder aufgefüllt werden konnten. ${ }^{27}$

Diese beiden Krisen zeigten nur zu deutlich, welches Ausmaß die Abhängigkeit der Schweiz und insbesondere ihrer Gaswerke von den Lieferungen der ausländischen

Marek, Kohle, S. 150-152.

Ebd., S. 153 f.

Ebd., S. 155-159.

27 Ebd., S. 159. 
Kohle hatte und wie wichtig funktionierende Transportrouten, aber auch ausreichende Lagerbestände für die Versorgungssicherheit waren.

\section{Konkurrenz durch elektrischen Strom}

Trotz wechselhaften, von Krisen beeinflussten Jahrzehnten konnte sich die Gasindustrie schließlich konsolidieren. Die Produktionsverfahren wurden fortwährend verbessert, die Gaswerke steigerten ihre Umsätze und konnten immer mehr Kunden anwerben - goldene Zeiten schienen bevorzustehen. Doch nun begann auch der Aufstieg der Elektrizität.

Elektrischer Strom wurde in der (Stadt-) Beleuchtung zum Hauptkonkurrenten des Gases. 1879 ließ der Unternehmer Johannes Badrutt den Speisesaal seines Hotels in St. Moritz mit elektrischem Strom beleuchten. Diese technische Sensation wurde von der Presse mit Begeisterung aufgenommen. ${ }^{28}$ Zwar war die elektrische Bogenlampe zu diesem Zeitpunkt eine noch recht neue Erfindung (Anlagen dieser Art hatten zunächst noch mehr den Charakter von "Spektakeln" und Prestigeprojekten), die Technologie hatte aber Einzug in die Schweiz gefunden und ihr Potential wurde auch vielerorts bereits erkannt. Im Folgejahr wurde das eidgenössische Sängerfest in Zürich mit unzähligen Bogenlampen illuminiert. Damit war die Leistungsfähigkeit der neuen Technologie bewiesen, das elektrische Licht verwandelte die Nacht quasi zum Tag. ${ }^{29}$

Die Kommunen waren nun in einer gewissen Zwickmühle. Sie hatten in den letzten Jahrzehnten große (finanzielle) Anstrengungen unternommen, die Gasbeleuchtung in ihren Städten und Gemeinden zu etablieren und auszubauen. Nun gab es eine neue, „bessere" Möglichkeit, die Beleuchtung der Straßen und Plätze zu gewährleisten. Sollten sie am Stadtgas festhalten oder doch auf die neue Technologie setzen? Für und Wider mussten abgewogen werden: Dagegen sprach etwa, dass eine funktionierende Beleuchtungsinfrastruktur bereits vorhanden war, viele der Gaswerke befanden sich schon im Besitz der Kommunen und warfen auch zum Teil gute Gewinne ab. Zudem war die Produktion relativ günstig. Argumente, die für eine Umstellung sprachen, waren u. a., dass das elektrische Licht als „sauberer" galt, heller leuchtete und keinen Ruß erzeugte. Die Erfahrungen der Kohleeinfuhr-Verknappung während der zuvor genannten Krisen zeigten auch die Abhängigkeit von ausländischen Importen auf, während Wasser für die Stromerzeugung in der Schweiz reichlich vorhanden war. Und nicht zuletzt war es auch eine Frage von Prestige, sich den Luxus elektrischen Lichts leisten zu können. ${ }^{30}$

Die Betreiber der Gaswerke mussten sich nun rasch auf diese neue Konkurrenz einstellen und neu positionieren. Die Straßenbeleuchtung ging bald an die Elektrizitätswerke verloren, dafür fand das Gas vermehrt in Betrieben und Haushalten Verwendung: als Energieträger für Heiz- und Kochzwecke. Dass sich das Gas hier so schnell etablieren 
konnte, liegt an mehreren Faktoren: Die Schweizer heizten vergleichsweise weniger mit Kohle als Konsumenten in anderen Ländern, was dem Faktum geschuldet war, dass diese für sie schwerer zugänglich und zudem generell teurer war. Auch der Umstand, dass die meisten Gaswerke mittlerweile kommunale Betriebe waren, war entscheidend. Die Städte sahen es als ihre infrastrukturelle Aufgabe an, möglichst alle Bewohner an das Gasnetz anzuschließen. Dabei übernahmen sie auch oftmals teilweise oder gänzlich die hierfür anfallenden Kosten. Auch kamen die Werke den Kunden oftmals entgegen und schlossen etwa an die gelegten Koch- und Heizgasleitungen auch Gaslampen unentgeltlich an. Dies führte dazu, dass die Errichtungskosten - wenn vorhanden - relativ gering waren und dass dadurch vielen Menschen ein Zugang zu dem Energieträger ermöglicht wurde. ${ }^{31}$ Zudem gewährten die Produzenten mancherorts auch beträchtliche Rabatte. Das hob etwa die St. Galler Zeitung „Die Ostschweiz” in ihrer Ausgabe vom 21. Jänner 1899 in einem Artikel besonders hervor:

„Bekanntlich wird die Verwendung des Leuchtgases zum Kochen, dank den mannigfachen Vorzügen, die sie gegenüber der Holz- und Kohlenfeuerung darbietet, immer allgemeiner. Dem einzigen Bedenken, das gegen dieselbe geltend gemacht werden kann, dem Kostenpunkt, hat unsere Gasfabrik durch Herabsetzen des Gaspreises für Kochzwecke bereits in verdankenswerter Weise Rechnung getragen."32

Neben diesen Services und Vergünstigungen führten auch Inserate in Zeitungen und Zeitschriften zum Anwachsen des Kundenstamms, Gasherde und -boiler wurden vielerorts beworben. Diese „Technisierung" des Haushalts hing damit zusammen, dass ab den 1890er-Jahren ein spürbarer Rückgang an Dienstboten zu verzeichnen war, die sich aufgrund anhaltend bescheidener Arbeitsverhältnisse nun in anderen Bereichen, etwa der Industrie oder in Büros, betätigten. ${ }^{33}$ Die Gasgesellschaften profitierten von dieser Entwicklung verständlicherweise, da gasbetriebene Innovationen die Haushaltstätigkeit in vielen Bereichen erleichterten. Indes waren die Elektrizitätswerke auch nicht untätig und warben selbst verstärkt um neue Abnehmer, was den Zuwächsen der gaswirtschaftlichen Konkurrenz jedoch keinen Abbruch tat. Vielmehr zeichnete sich

„[...] überhaupt eine gewisse Arbeitsteilung in der Versorgung des Publikums mit Kraft und Licht einerseits und mit Wärme anderseits [ab], indem jene Aufgabe mehr von den Elektrizitätswerken, diese mehr von den Gaswerken beansprucht [wurde.]"34

31 o. A., Die Entwicklung der schweizerischen Gaswerke in den letzten zwanzig Jahren, in: Schweizerische Bauzeitung 35/36 (1900), Heft 26, S. 255-256, hier S. 255.

32 Dr. Kaiser, Das Warmwasserschiff für Gasherde, in: Die Ostschweiz, Nr. 17, 21.1.1899, S. 6, [http://bit.ly/1HIRKhd], eingesehen 1.11.2015

33 Ines Siegfried Schnider, „Hausfrauen kocht elektrisch!“ Das Eindringen von Elektroherd und elektrischen Geräten in die städtische Küche der Zwischenkriegszeit, in: David Gugerli (Hrsg.), Allmächtige Zauberin unserer Zeit. Zur Geschichte der elektrischen Energie in der Schweiz, Zürich 1994, S. 155-165, hier S. 155. 
Gas- und Elektrizitätswirtschaft hatten sich ihre Bereiche vorerst abgesteckt. Den Gaswerken kam hier natürlich auch die Tatsache zu Gute, dass die Verwendung von elektrischem Strom noch einige Zeit deutlich teurer sein sollte als die von Gas. ${ }^{35}$

Die Wachstumsraten beim Gasabsatz (siehe dazu Abbildung 1 im Anhang) nahmen in der gesamten Schweiz noch bis zum Ersten Weltkrieg beständig zu. Diese Entwicklung glich in der Schweiz in weiten Teilen jener im Deutschen Reich. ${ }^{36}$

\section{Die Gasindustrie während des Ersten Weltkriegs}

Wie schon zuvor beschrieben, war die schweizerische Gasindustrie abhängig von Kohleimporten aus dem Ausland - etwa aus dem Deutschen Reich oder Frankreich. Als diese beiden Länder im Sommer 1914 (erneut nach 1870) in den Kriegszustand traten, lag die Vermutung nahe, dass dies (erneut) negative Auswirkungen auf die Kohleeinfuhren der Schweiz haben würde. Dem war jedoch nicht so - zumindest nicht sofort: Noch bis in das Jahr 1916 hinein konnten die Gaswerke Produktionssteigerungen verzeichnen. Dieses vermeintliche Paradoxon kann jedoch schnell erklärt werden: Der Krieg erschwerte den Import von flüssigen Brennstoffen wie Benzin oder Petrol - das Gas musste diese Lücke schließen. ${ }^{37}$

Dass es bei dem benötigten Vergasungsrohstoff anfangs keine Engpässe gab, war einer Genossenschaft zu verdanken. Bereits 1910 hatte sich die Mehrzahl der schweizerischen Gaswerke zu einer Einkaufsvereinigung zusammengeschlossen, um so vereint am internationalen Kohlemarkt auftreten und den Import zu den besten Konditionen verhandeln zu können. Deren noch in den Vorkriegsjahren abgeschlossenen Verträgen war es zu verdanken, dass die Einfuhren in den ersten gut anderthalb Jahren des Weltkriegs noch zu relativ guten Konditionen erfolgten. Trotz einiger Rückschläge, wie etwa verringerter Transportkapazitäten oder zeitweilig gültiger Kohleexportverbote seitens der deutschen Reichsregierung, konnten die Gaswerke ihren Betrieb mit Ausnahmen fortführen. ${ }^{38}$

Im Laufe des Jahres 1916 verschlechterten sich die Rahmenbedingungen dann jedoch merklich. Die Lieferverträge waren großteils ausgelaufen und die Preise stiegen in Folge massiv an, die Importe waren jetzt kaum noch leistbar. Schweizer Regierung und Gasproduzenten mussten sich auf ein weiteres Vorgehen einigen. Maßnahmen sollten gesetzt werden, um den Gaskonsum zu reduzieren. Die Situation verschärfte sich dennoch, einige Werke waren gar darauf angewiesen, alternative Rohstoffe wie Holz oder Torf für die Vergasung zu verwenden. Daraufhin verschlechterte sich die Gasqualität merklich. Die Verknappung und der verminderte Brennwert führten dazu, dass die

35 Gugerli, Redeströme, S. 49.

36 Dieter Schott, Europäische Urbanisierung (1000-2000). Eine umwelthistorische Einführung, Stuttgart 2014, S. $286 \mathrm{f}$.

37 Christoph Menasse, Strukturwandel und Neuorientierung der Gasindustrie in der Zwischenkriegszeit unter Berücksichtigung des Gaswerks Basel, in: Basler Zeitschrift für Geschichte und Altertumskunde 105 (2005), S. 49-78, hier S. $51 \mathrm{f}$. 
Gaswerke erstmals rückläufige Abonnentenzahlen verzeichnen mussten. ${ }^{39}$ Im Gegenzug konnten sich die Elektrizitätsgesellschaften über zahlreiche Neukunden freuen, da sie im Krieg weitgehend auf Tariferhöhungen verzichteten und ihr Strom dadurch im Vergleich zu Gas immer konkurrenzfähiger wurde. ${ }^{40}$ Es sollte noch bis weit in die Zwischenkriegszeit hinein dauern, bis sich die Gaswirtschaft von diesen Entwicklungen erholen konnte. ${ }^{41}$

Um die zuvor beschriebenen Entwicklungen exemplarisch darstellen zu können, soll im folgenden Kapitel abschließend die Geschichte der Zürcher Gasindustrie skizziert werden, die sich ab den 1890er-Jahren zur mit Abstand leistungsfähigsten der gesamten Schweiz entwickelt hatte. ${ }^{42}$

\section{Die Gaswerke der Stadt Zürich}

Angesichts der wachsenden Bevölkerung und dem steigenden Bedürfnis nach einer adäquaten Straßenbeleuchtung entschied der Zürcher Stadtrat um die Mitte des 19. Jahrhunderts, dass die bestehende Ölbeleuchtung sowohl im Betrieb, als auch angesichts ihrer Leuchtleistung nicht mehr zeitgemäß war und beschloss, zukünftig ebenso auf Stadtgas zu setzen. Zudem konnte man durch das Abgeben von Errichtung und Betrieb mögliche finanzielle Risiken für die Stadt minimieren. Nach den Erfahrungswerten anderer Städte würde man sich jedoch dank strikter Vorgaben bezüglich Leuchtkraft, Leistung und Preis eine gewissen Kontrolle über das Gaswerk erhalten können. Für den Betrieb sollte Holz als zu vergasender Rohstoff verwendet werden, da man bei Kohlegas gesundheitsschädlichere Dünste fürchtete. Worauf diese Bedenken fußten, ist nicht zu eruieren, vermutlich auf dem wahrnehmbaren Schwefelgeruch. ${ }^{43}$

Im Jahr 1856 nahm dann das erste Gaswerk der Stadt, errichtet von der „Züricher Actiengesellschaft für Gasbeleuchtung", seinen Betrieb auf. Dem zugrunde lag eine Konzession, die auf dreißig Jahre ausgelegt war. Nach Ablauf dieser Frist hatte die Stadt die vertraglich zugesicherte Option, das Gaswerk von seinen privaten Betreibern abzulösen. Im Jahr 1862 versorgte die Anlage 604 Straßenflammen und belieferte 672 Privatabnehmer. ${ }^{44}$

Neben dem Stammwerk wurden nach 1867 zwei weitere Anlagen errichtet, die jedoch schon mit Kohle betrieben wurden. Diese konnte man über die neue Bahnlinie zwischen Basel und Zürich aus dem deutschen Rheinland nun leichter importieren. ${ }^{45}$ Gegen den bisher verwendeten Rohstoff Holz sprachen die bis dahin gewonnenen Erfahrungswerte. Das Holzgas konnte qualitativ nicht überzeugen, zu groß waren die

39 Menasse, Strukturwandel, S. $53 \mathrm{ff}$.

40 Gugerli, Redeströme, S. 15.

41 Menasse, Strukturwandel, S. $76 \mathrm{f}$.

42 Siehe Grafik im Anhang.

43 Marek, Kohle, S. $112 \mathrm{f}$.

44 N. H. Schilling, Statistische Mittheilungen über die Gas-Anstalten Deutschlands, der Schweiz und einige GasAnstalten anderer Länder, München 1868, S. 369, [http://reader.digitale-sammlungen.de/de/fs1/object/display/ bsb10305779_00379.html], eingesehen 1.11.2015. 
Schwankungen der chemischen Zusammensetzung. Ebenso war es schwierig, Abgabemengen konstant zu halten, da sich das Gasvolumen bei Temperaturveränderungen deutlich änderte. ${ }^{46}$

Die Lieferschwierigkeiten während des Deutsch-Französischen Krieges 1870/71 trafen das Unternehmen schwer. Die Kohlebestellungen konnten aus dem Saarland nicht nach Zürich transportiert werden, die öffentliche Beleuchtung musste reduziert werden und schließlich war man gezwungen, für die Vergasung nur unzureichend taugliche Kohle aus dem französischen Loirebecken zu importieren. ${ }^{47}$

Nach Ablauf der Konzession übernahm die Stadt die drei Gaswerke und ergänzte die nun kommunale Gesellschaft um einen weiteren Standort. ${ }^{48}$ Zürichs Bevölkerung wuchs - nicht zuletzt auch aufgrund von Eingemeindungen im Jahr 1893 - und somit stieg auch der Bedarf an Stadtgas kontinuierlich an. Dazu kam, dass sich in den 1890erJahren auch die Abnehmerstrukturen änderten. Waren zuvor noch die städtische und private Beleuchtung Hauptverbraucher des Gases, wurde es in diesem Bereich nach und nach von der aufkeimenden Elektrizität verdrängt. Doch die bereits beschriebene alternative Vermarktung war auch hier schnell gefunden - die Verwendung als Kochund Heizgas in Privathaushalten. Der Gaskonsum stieg folglich deutlich an, eine Expertise rechnete mit einer durchschnittlichen Zunahme von zwölf Prozent pro Jahr. ${ }^{49}$

Vor diesem Hintergrund entschied sich die Stadt 1894, den Bau einer Großanlage in Auftrag zu geben. Zunächst musste ein geeignetes Bauland gefunden werden, das etwa noch Platz für etwaige spätere Erweiterungen bot, sich nahe einer Bahnlinie und auf einem tieferen Niveau befand, um die Weiterleitung des Gases zu den Abnehmern gewährleisten zu können. Man entschied sich für ein Gelände in Schlieren, unweit von Zürich gelegen. Im nächsten Schritt wurde die nötige Kapazität berechnet, die das Werk in Zukunft leisten sollte. Ebenso wurde an Lagerstätten gedacht, um im Krisenfall ausreichend Kohlereserven vorrätig zu haben. Der erste Teil des Gaswerks Schlieren nahm dann bereits 1898 den Betrieb auf. Die Tagesleistung betrug anfänglich mindestens $25.000 \mathrm{~m}^{3}$, die in drei Teleskopgasometern zwischengelagert werden konnten. ${ }^{50}$

Schon bald erkannte man, dass die anfänglichen Prognosen zum ansteigenden Konsum zu kurz gegriffen waren. Während die Zürcher Bevölkerung zwischen 1899 und 1909 nur um gut 3,5 Prozent pro Jahr zunahm, stieg der Gasverbrauch jedoch um rund vierzig Prozent jährlich. Schon bald würden die Produktionskapazitäten nicht mehr ausreichen. Zwischen 1903 und 1909 wurde das Werk Schlieren um eine Ofenanlage mit einer Mindestleistung von $50.000 \mathrm{~m}^{3}$ ergänzt, das Kohlelager wurde vergrößert und ein weiterer Gasometer errichtet..$^{51}$ 
Bis in das Krisenjahr 1916 stieg die Produktion auf bis zu $156.000 \mathrm{~m}^{3} / 24 \mathrm{~h}$ und überschritt damit sogar die errechnete Maximalkapazität deutlich. Der bereits beschriebene kriegsbedingte Einbruch des Konsumvolumens traf auch das Zürcher Werk schwer. Erst zehn Jahre später war die Produktion wieder voll ausgelastet, was einen neuerlichen Zubau nötig machte. ${ }^{52}$

\section{Resümee}

Die Geschichte des Stadtgases in der Schweiz ist eine überaus wechselvolle. Nachdem es etwas länger als in den benachbarten Staaten gedauert hatte, bis die ersten Gaswerke gegründet worden waren, entwickelte sich jedoch rasch eine leistungsfähige und schnell wachsende Industrie, womit die Schweiz anfängliche Rückstände bald aufholen konnte. Wie auch jenseits der Grenze üblich, wurden die ersten Gaswerke von privaten oder halböffentlichen Unternehmen errichtet und betrieben, die Kommunen konnten sich des Risikos eines möglichen Scheiterns der Unternehmung weitgehend entziehen. Mit der Zeit wurden die meisten Betriebe jedoch von der öffentlichen Hand übernommen und weitergeführt. Gründe hierfür waren etwa fehlende Investitionen oder Kapital seitens der Privaten oder auch schon zuvor vertraglich zugesicherte Kaufoptionen zu Gunsten der Kommunen. Damit konnten diese auch für eine Verbesserung der Versorgung ihrer Bevölkerung Sorge tragen, indem sie etwa Anschlusskosten zum Teil übernahmen und leistbare Preise verlangten.

Auch in der Schweiz wurde Stadtgas gegen Ende des 19. Jahrhunderts mit der aufkommenden Elektrizität konfrontiert. Hier kam es aber in der Folge zu einer Aufteilung der Absatzgebiete: Während die Straßen fortan zumeist mit Strom erhellt wurden, eroberte Stadtgas die Privathäuser und Betriebe als Energieträger für Heiz- und Kochzwecke.

Mit der Erschließung dieses neuen Bereichs stiegen auch die Absatzzahlen von Jahr zu Jahr an. Was den schweizerischen Gaswerken jedoch stets zum Verhängnis werden konnte und auch wurde, war die große Abhängigkeit von ausländischen Kohlelieferungen. Erstmals mussten sie das im Deutsch-Französischem Krieg 1870/71 erfahren, als die Zufuhr nicht mehr funktionierte und die Reserven bald aufgebraucht waren. Ebenso gravierend wirkten sich die Bergarbeiterstreiks am Beginn der 1890er-Jahre aus. Der Weltkrieg hatte indes in seinen ersten beiden Jahren keine derart drastischen Folgen für die Gasproduzenten, die sich mit langfristigen Lieferabkommen diesmal besser abgesichert hatten. Doch auch diese konnten keine dauerhafte Lösung gewährleisten. 1916 liefen die meisten der Verträge aus, was zu einer erneuten Versorgungskrise führte, an deren Folgen die Gasindustrie noch bis weit in die Zwischenkriegszeit zu leiden hatte.

Zürich war nicht unter den ersten Schweizer Städten, die sich eine Gasbeleuchtung leisten wollten. Trotzdem verlief der Einstieg erfolgreich, die Werke konnten den steigenden Bedarf schon bald nicht mehr decken und die Kapazität wurde beständig er- 
weitert. In den 1890er-Jahren wurde schließlich mit dem Gaswerk Schlieren ein Projekt initiiert, das den Ansprüchen einer wachsenden Großstadt gerecht werden konnte.

Die oben beschriebenen Krisen trafen auch die Zürcher Gasindustrie zum Teil schwer, insbesondere der Erste Weltkrieg. Erst eine gute Dekade nach Kriegsende konnten die Konsumwerte des Jahres 1916 wieder erreicht werden.

\section{Quellen}

o. A., Die Entwicklung der schweizerischen Gaswerke in den letzten zwanzig Jahren, in: Schweizerische Bauzeitung 35/36 (1900), Heft 26, S. 255-256.

Kaiser, Dr., Das Warmwasserschiff für Gasherde, in: Die Ostschweiz, Nr. 17, 21.1.1899, S. 6, [http://bit.ly/1HIRKhd], eingesehen 1.11.2015.

Ott, E., Die schweizerischen Gaswerke, in: Schweizerische Bauzeitung 65/66 (1915), Heft 14, S. 156-158.

Pfeiffer, Otto, Das Gas als Leucht-, Heiz- und Kraftstoff in seinen verschiedenen Arten als Steinkohlegas, Holz- und Torfgas, Oelgas, Wassergas. Fabrikation und Verwendung nach dem neuesten Standpunkt, unter Berücksichtigung der Konkurrenzverhältnisse zwischen Gas und Elektrizität, Zum 100-jährigen Jubiläum der Gasindustrie, Weimar 1896.

Schilling, N. H., Statistische Mittheilungen über die Gas-Anstalten Deutschlands, der Schweiz und einige Gas-Anstalten anderer Länder, München 1868, S. 369, [http://rea der.digitale-sammlungen.de/de/fs1/object/display/bsb10305779_00379.html], eingesehen 1.11.2015.

\section{Literatur}

Gasversorgungsunternehmen in Berlin, Findbuch. Landesarchiv Berlin, Bestandsgruppe A Rep. 259, [http://www.landesarchiv-berlin.de/php-bestand/arep259-pdf/arep259. pdf], eingesehen am 1.11.2015.

Grunder, Karl, Die Kunstdenkmäler des Kantons Zürich. Band IX Der Bezirk Dietikon (Die Kunstdenkmäler der Schweiz 88), Basel 1997.

Gugerli, David, Redeströme. Zur Elektrifizierung der Schweiz 1880-1914, Zürich 1996.

Hutchinson, Kenneth, The Royal Society and the Foundation of the British Gas Industry, in: Notes and Records of the Royal Society of London 39 (1985), No. 2, S. 245-270.

Jüptner, Hans von, Wärmetechnische Grundlagen der Industrieöfen. Eine Einführung in die Wärmelehre und gedrängte Übersicht über die verschiedenen Arten von Brennstoffen und ihre Verwertung, Berlin 1927.

Kretschmer, Helmut, Gasometer in Wien. Industrie- und Technikdenkmale im Wandel der Zeit (Wiener Geschichtsblätter Beiheft 1/2001), Wien 2001. 
Langbein, Hans Peter, Die schweizerische Steinkohlengasindustrie an der Schwelle des Atomzeitalters, phil.-hist. Diss. Basel 1961.

Marek, Daniel, Kohle. Die Industrialisierung der Schweiz aus der Energieperspektive 1850-1900, phil.-hist. Diss. Bern 1992.

Menasse, Christoph, Strukturwandel und Neuorientierung der Gasindustrie in der Zwischenkriegszeit unter Berücksichtigung des Gaswerks Basel, in: Basler Zeitschrift für Geschichte und Altertumskunde 105 (2005), S. 49-78.

Schivelbusch, Wolfgang, Lichtblicke. Zur Geschichte der Helligkeit im 19. Jahrhundert, Frankfurt am Main 2004.

Schott, Dieter, Europäische Urbanisierung (1000-2000). Eine umwelthistorische Einführung, Stuttgart 2014

Siegfried Schnider, Ines, „Hausfrauen kocht elektrisch!“ Das Eindringen von Elektroherd und elektrischen Geräten in die städtische Küche der Zwischenkriegszeit, in: David Gugerli (Hrsg.), Allmächtige Zauberin unserer Zeit. Zur Geschichte der elektrischen Energie in der Schweiz, Zürich 1994, S. 155-165.

The National Archives, Chartered Gas Light and Coke Company, o. D. [http://discovery. nationalarchives.gov.uk/details/rd/3c478014-712e-43c6-8786-c3b871813664], eingesehen 2.12.2015.

\section{Weiterführende Literatur}

Barty-King, Hugh, New flame. How gas changed the commercial, domestic and industrial life of Britain between 1813 and 1984, Tavistock-Devon 1984.

Dax, Ingo, Von Stadtgas- auf Erdgasbetrieb. Die Umstellung in der Industrie von Stadtgas- auf Erdgasbetrieb, in: Schweizer Maschinenmarkt 72 (1972), Heft 49, S. 80-83.

Hodel, Fabian, Vom Leuchtgas zur modernen Energie. 100 Jahre Gaswerk der Stadt Luzern, 1895-1995, Luzern 1995.

Paquier, Serge, From the Following to Improving Technology. The Case of the Swiss Gas Industry in the $19^{\text {th }}$ Century, in: Quaderns d'Història de l'Enginyeria 12 (2011), S. 171-199.

Paquier, Serge/Williot, Jean-Pierre (Hrsg.), L'industrie du gaz en Europe aux XIXe et XXe siècles. L'innovation entre marchés privés et collectivités publiques (Euroclio 20), Brüssel 2005.

Peebles, Malcolm W. H, Evolution of the gas industry, London 1980.

Thau, Adolf, Die Stadtgasindustrie. Ein Abriss ihrer geschichtlichen Entwicklung, Berlin 1935 


\section{Anhang}

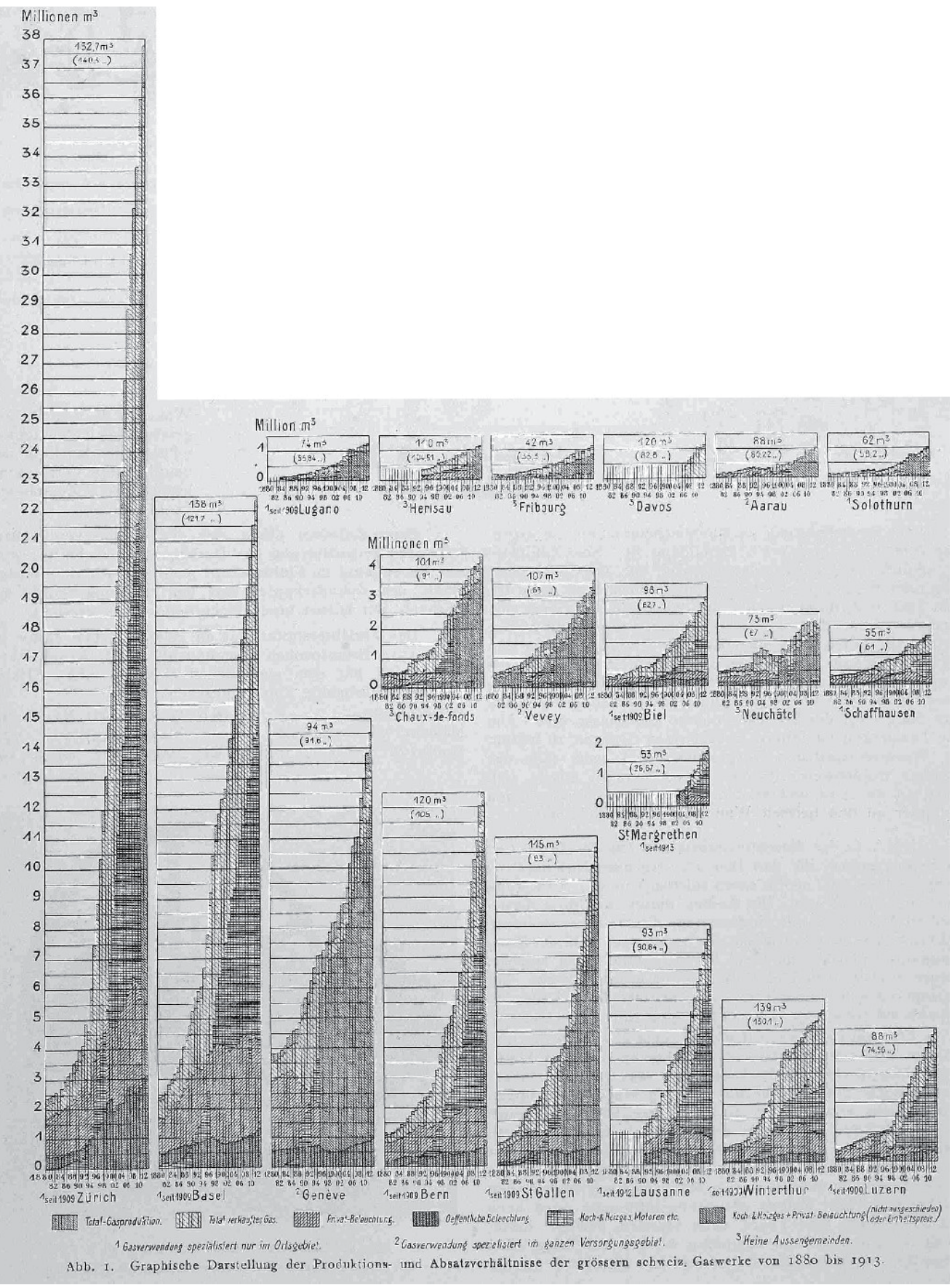

Abbildung 1 Graphische Darstellung der Produktions- und Absatzverhältnisse der größeren schweizerischen Gaswerke von 1880 - 1913, publiziert in: E. Ott, Die schweizerischen Gaswerke, in: Schweizerische Bauzeitung 65/66 (1915), Heft 14, S. 156-158, hier S. 156. 
Maximilian Oswald ist Studierender der Geschichtswissenschaften (Master) im 2. Semester und studentischer Mitarbeiter am Institut für Geschichtswissenschaften und Europäische Ethnologie (Kernfach Wirtschafts- und Sozialgeschichte) an der Universität Innsbruck. maximilian.oswald@student.uibk.ac.at

\section{Zitation dieses Beitrages}

Maximilian Oswald, Gas erleuchtet die Schweiz. Stadtgas als Energieträger des 19. und frühen 20. Jahrhunderts, in: historia.scribere 8 (2016), S. 391-408, [http://historia.scribere.at], 2015-2016, eingesehen 14.6.2016 (=aktuelles Datum).

(C) Creative Commons Licences 3.0 Österreich unter Wahrung der Urheberrechte der Autorlnnen. 
